\title{
Engineered embryonic stem cells for regnerative nedicine
}

\author{
$\mathrm{J}^{\text {Hescheler }}{ }^{1}$ \\ ${ }^{1}$ Institut für Neurophysiologie, Robert-Koch-Str. 39, 50931 Köln, Germany
}

Due to their ability to reproduce the embryological differentiation of nearly all different cellular phenotypes, embryonic stem (ES) cells represent an ideal tool to study processes of embryogenesis under in vitro conditions, in particular the signalling cascades and genes involved in the functional development (functional genomics), as well as to provide a new source for cellular replacement therapy. We cultivated ES cells in three dimensional cell aggregates (embryoid bodies), where they differentiate into derivatives of all three germ layers. To select only one lineage, e.g. the cardiac lineage, and to allow the identification of the transplanted cells, transgenic ES cells were used. They contained a bicistronic vector with two cloning sites for EGFP and an antibiotics resistance for selective selection both under the $\alpha$-MHC promoter. To demonstrate the ability of ES cells for regenerative medicine and tissue repair, cardiomyocytes differentiated from ES cells were injected into the cryoinfarcted left ventricular wall of adult wild type mice. Immunological cross reactions were avoided by using the same inbred mouse strain. EGFP positive transplanted cardiomyocytes could easily be detected in the native heart at different intervals after operation. The cells were found to engraft and to differentiate into adult-like cardiomyocytes as confirmed by cross striation after immunostaining with a-actinin. These data were corroborated by patch clamp experiments on isolated EGFP positive cardiomyocytes at different time points after operation. The transplanted cells displayed ventricular action potentials and $\beta$-adrenergic- as well as muscarinic regulation. When survival was investigated in a large colony of mice, the control group had an almost double mortality rate as compared to the transplanted mice. Our data show engraftment and differentiation of embryonic cardiomyocytes after transplantation into cryoinfarcted areas of the heart. Before a clinical use of human ES cells for therapeutic trials in humans two major prerequisites must be fulfilled: (i) they must be safe, i.e. the development of tumours because of the high proliferative potential must be omitted and (ii) the rejection of the transplanted cells must be prevented. For the criterion (i) the technology of "lineage selection" appears optimal, for the criterion (ii) reprogramming techniques are now tested. Although autologous bone marrow cells appear ideal to circumvent rejection, we were not able to demonstrate a transdifferentiate into cardiomyocytes. Hence emphasis is made on reprogrammed human ES cells. The complexity of questions to be answered makes it necessary to establish closer collaborations between various disciplines including cell-, molecular-, developmentalbiology, molecular genetics, biochemistry, immunology, as well as clinical disciplines with medical basic sciences, experimental surgery and bioengineering together with cell culture techniques and material sciences.

Cell Research (2008) 18:s111. doi: 10.1038/cr.2008.201; published online 4 August 2008

Prof Jürgen Hescheler $(\mathrm{PhD})$ is Chairman and Director of the Institute of Neurophysiology at the Universität zu Köln. He has been working with embryonic stem cells of the mouse for over 18 years. Beginning with studies on cellular signal transduction, he has defined many important basic aspects both of fundamental research and of clinical applications. He was the first scientist worldwide to perform electrophysiological experiments on stem cells thus pioneering the establishment of stem cell research for application in transplantation medicine and in 2003 he was given the one of the first permissions to work with human embryonic stem cells in Germany. In 2005 he founded the German Society for Stem Cell Research. 